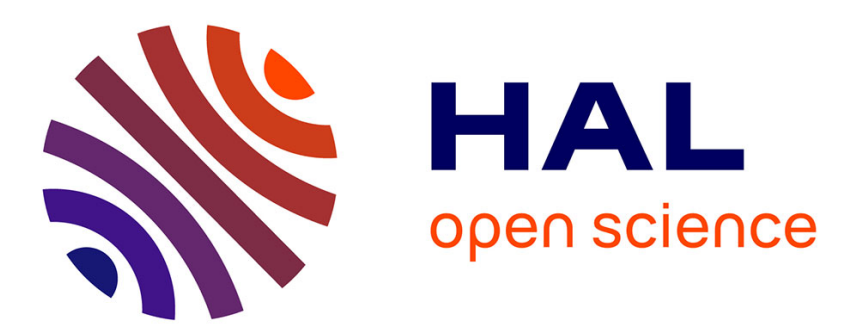

\title{
Stratégies informationnelles en milieu professionnel: du réseau personnel à la Toile
}

Céline Paganelli, Evelyne Mounier

\section{To cite this version:}

Céline Paganelli, Evelyne Mounier. Stratégies informationnelles en milieu professionnel: du réseau personnel à la Toile. Tracer l'horizon informationnel du XXIe siècle: Frontières, passerelles et carrefours: 37ème congrès annuel de l'Association Canadienne des Sciences de l'Information., May 2009, Carleton University, Ottawa, Ontario., Canada. 18p. hal-00652112

\section{HAL Id: hal-00652112 \\ https://hal.science/hal-00652112}

Submitted on 14 Dec 2011

HAL is a multi-disciplinary open access archive for the deposit and dissemination of scientific research documents, whether they are published or not. The documents may come from teaching and research institutions in France or abroad, or from public or private research centers.
L'archive ouverte pluridisciplinaire HAL, est destinée au dépôt et à la diffusion de documents scientifiques de niveau recherche, publiés ou non, émanant des établissements d'enseignement et de recherche français ou étrangers, des laboratoires publics ou privés. 
Céline PAgANELLI

Evelyne MOUNIER

GRESEC, Université Stendhal Grenoble III

Institut de la Communication et des Médias

11, Avenue du 8 mai 1945 - 38130 Échirolles

\title{
Stratégies informationnelles en milieu professionnel: du réseau personnel à la Toile
}

Résumé : Cette communication s'intéresse au lien entre l'expertise des individus, le contexte de la tâche et les stratégies de recherche d'informations dans un cadre professionnel. Des entretiens, menés auprès d'employés d'une banque, montrent que les types de recherche et le choix des ressources sont conditionnés par les objectifs et les contraintes liés à l'activité de l'individu mais aussi à l'activité de l'entreprise.

\begin{abstract}
:

\section{Introduction}

Les modes de recherche d'information, les pratiques et usages de l'information sont largement étudiés depuis longtemps; enfants et adultes, grand public, étudiants ou chercheurs ont fait l'objet de nombreuses observations. En revanche, l'intérêt pour les pratiques du monde professionnel (industriel, commercial, médical, etc.) est plus récent et peu de travaux se sont intéressés à l'incidence de l'activité et du contexte professionnel sur la recherche d'information (Jarvelin, 2004). Comment s'informe-t-on dans un contexte professionnel ? A quelles ressources s'adresse-t-on et comment les sélectionne-t-on à l'heure où il est courant de dire que tout est disponible sur la Toile? Les exigences en information professionnelle conduisent-elles à des pratiques et des usages particuliers?
\end{abstract}


L'étude proposée porte sur le lien entre l'expertise des individus, le contexte de la tâche et les stratégies de recherche d'informations dans un cadre professionnel. Nous considérons que la recherche d'information proprement dite n'est qu'une stratégie parmi d'autres, destinées à obtenir l'information utile. Nous montrerons que les types de recherche ainsi que le choix des ressources sont conditionnés par les objectifs et les contraintes liés à la fonction et aux tâches de l'individu dans l'entreprise. De même, nous montrerons que c'est l'expertise qui favorise l'accès aux sources formelles parce que les experts disposent des connaissances nécessaires.

\section{1- L'influence du contexte sur les pratiques d'information en milieu professionnel}

De très nombreux facteurs peuvent affecter le déroulement d'une recherche d'informations comme l'âge, la connaissance $\mathrm{du}$ fonctionnement $\mathrm{du}$ système ou encore les connaissances préalables. D'autres facteurs peuvent intervenir : ainsi, Kulthau (Kulthau 2004) a montré que les états affectifs ont une incidence sur la recherche d'information ; d'autres auteurs (Guyot, 2000) (Jarvelin, 2004) ont également souligné l'importance du contexte professionnel et des contraintes spécifiques de la tâche. Ainsi, la recherche d'information est également affectée par le contexte et l'activité à mener (Bartlett, 2005) (Guyot, 2002).

\section{1-1- La spécificité du contexte professionnel}

En termes d'accès à l'information, le contexte professionnel constitue un contexte particulier. L'activité d'information, entendue comme l'ensemble des démarches mises en œuvre pour s'informer dans le but de mener à bien son activité professionnelle, prend une place de plus en plus importante et se complexifie (Guyot, 2002), notamment en raison de la multiplication des sources d'information disponibles et du développement d'outils nécessitant des compétences spécifiques pour leur utilisation. Accart (Accart, 2002) remarque que les activités professionnelles sont largement fondées sur l'échange d'informations et de connaissances. En fait, l'activité d'information est constitutive de chaque activité professionnelle, quel que soit le poste ou la fonction occupée. Pourtant, une 
enquête menée auprès d'une dizaine d'entreprises en RhôneAlpes montre que, dans la plupart des cas, les pratiques d'information sont très hétérogènes, peu formalisées et peu développées dans les entreprises observées. Négligence, manque de moyens, peu d'importance accordée à l'information, à la culture de l'information, sont des explications avancées lors des entretiens menés par Accart (Accart, 2002). Cette activité d'information semble liée à la culture informationnelle de l'entreprise (Bergeron, 2007) (Choo, 2007). Ainsi l'importance que l'entreprise accorde à la place de l'information influencerait les pratiques informationnelles des individus.

L'activité d'information est enfin contrainte par des exigences particulières, spécifiques au contexte professionnel : «elle prend place dans le cadre général de l'activité, plus ou moins fortement contraint par des objectifs et des échéances » (Guyot, 2002). Quand un individu en situation de travail a besoin d'une information pour poursuivre sa tâche, les exigences de rapidité d'obtention de l'information et de précision des résultats obtenus sont souvent très fortes.

\section{1-2- L'influence de la tâche et de l'expertise sur les pratiques d'information}

Le déroulement même d'une tâche conditionnerait les modes d'accès à l'information. Ainsi, Cheuk (Cheuk, 1999) montre, que chez des ingénieurs en situation de travail, les stratégies d'accès à l'information varient selon les étapes de la tâche à effectuer. L'auteur met en évidence sept situations de recherche et d'utilisation de l'information tout au long du déroulement de la tâche. Il fait un lien entre ces situations et les comportements informationnels (choix des sources / jugement de pertinence de l'information / stratégie d'organisation de l'information / sensations / définition de l'information). Par exemple concernant le choix des sources d'information, lors de la $1^{\text {ère }}$ phase du déroulement de l'activité (formulation de la tâche à mener si elle est nouvelle), les ingénieurs ont tendance à utiliser des sources facilement accessibles (presse, internet) alors que dans les phases de confirmation ou de vérification les ingénieurs se tournent vers des sources spécialisées qui font autorité. 
S'agissant de l'expertise professionnelle, la question est complexe, et ce pour plusieurs raisons. Tout d'abord, la notion d'expertise elle-même est controversée (Olmstadt, 2000). Pour Hung (Hung, 2003), l'expertise concerne les connaissances et l'expérience que les individus mettent en œuvre pour prendre des décisions dans des situations complexes. Selon Ericsson (Ericsson, 1997), il faut plusieurs années d'activités régulières et répétées pour que s'installe une véritable expertise dans un domaine précis. L'expertise présenterait donc les caractéristiques suivantes : spécifique à un domaine, elle serait acquise par étapes et représenterait un mélange d'analyse et d'intuition; ainsi, l'expert serait celui qui a mis en place des raisonnements heuristiques, lui permettant de résoudre rapidement et efficacement des problèmes.

Par ailleurs, dans toute situation professionnelle et notamment en situation de recherche d'information, plusieurs types d'expertise peuvent intervenir : l'expertise dans le domaine, dans l'entreprise, ou encore dans l'utilisation du système d'information. Enfin, intuitivement, un expert pourrait être un usager n'ayant pas besoin de rechercher des informations en raison de son expérience et de sa maitrise de l'activité qu'il a en charge. Pourtant, on sait qu'il n'en est rien puisque experts et novices utilisent des ressources d'information mais leurs besoins sont différents : les experts tendent à rechercher des informations ponctuelles avec des objectifs de vérifications le plus souvent, alors que les novices recherchent des connaissances plus générales parce qu'ils sont engagés dans un processus d'apprentissage (Paganelli et Mounier, 2003). L'expertise influe également sur les stratégies de recherche d'information, sur le temps passé à la formulation d'une requête, ou au traitement des réponses obtenues. (Dinet, 2002), (Marchionnini, 1990)

\section{2- Les stratégies d'information au sein d'une entreprise bancaire}

\section{2-1- Objectifs et méthodologie}

L'étude se situe dans le cadre d'une entreprise bancaire. Deux populations ont été étudiées : les commerciaux et les 
contrôleurs. Les commerciaux sont affectés à l'accueil de la clientèle. Ce sont eux qui répondent aux questions des clients et procèdent à l'ouverture des comptes. Les chargés de contrôle exercent leur activité en «back office». Ils sont chargés de vérifier la validité des dossiers et d'intervenir dans les cas litigieux. Pour chacune de ces catégories, 2 types de sujets ont participé à l'étude : des experts ayant plus d'un an d'expérience sur le poste, et des novices en charge depuis moins d'un an de l'activité concernée.

Une tâche pilote a été choisie, désignée par le terme «d'Entrée en Relation » ou EER. Elle est à la base de toute l'activité des agents d'une entreprise bancaire. Elle recouvre à la fois l'accueil des clients réguliers, la prise de contact avec les nouveaux clients, l'ouverture d'un dossier d'ouverture de compte, les demandes éventuelles de prêts, la vente de services et produits bancaires. Elle est aujourd'hui très encadrée et contrôlée par le système d'information. Par ailleurs, la législation française a rendu les banques responsables devant la loi, des opérations et des transactions qu'elles acceptent pour leurs clients. De ce fait, ces entreprises sont amenées à durcir les mesures de contrôles. La formation et l'information des employés de l'entreprise sont donc primordiales.

L'analyse des entretiens porte sur deux points : la manière dont ces deux groupes de professionnels décrivent le déroulement de cette tâche et les demandes d'information qu'ils sont amenés à formuler tout au long de son déroulement; et la manière dont ils recherchent et obtiennent ou non l'information souhaitée.

Les entretiens ont été conduits de manière à faire expliciter par une personne une série d'actions plus ou moins planifiées, pour déterminer quels sont les besoins récurrents en information et comment ces besoins sont formulés. Ils ont duré environ 1 heure. 23 employés de la banque ont été rencontrés. Ils assument les fonctions suivantes : commerciaux (13), contrôleurs (10). 17 d'entre eux sont experts dans leurs fonction (ils l'assument depuis plus d'un an), les 6 autres sont novices.

\section{2-2- Analyse et résultats}


Trois types d'informations ont été recueillis suite aux entretiens : d'abord des informations ayant trait à l'organisation de la tâche d'EER en une série de sous-tâches, ensuite des exemples de besoins d'informations intervenant tout au long du déroulement de la tâche, enfin les stratégies mises en œuvre pour obtenir les informations.

\section{2-2-1- L'influence de la tâche sur le type d'information attendue}

\subsubsection{Organisation de la tâche}

Pour l'ensemble des sujets, la tâche d'EER se décompose en quatre sous tâches qui peuvent être listées comme suit :

o Présentation des produits, de l'entreprise et des règles à suivre pour une ouverture de compte ;

o Demande de pièces justificatives et demande d'information auprès du client sur ses attentes ;

o Vérification de chaque pièce du dossier ;

o Vérification du dossier complet.

Ces sous tâches peuvent être regroupées autour de deux pôles principaux : celui de l'interaction avec le client qui est soumis à des exigences commerciales et relationnelles, et celui du respect de la réglementation. Un troisième pôle est apparu lors des entretiens, il concerne les aspects informatiques liés à la mise en œuvre de la tâche.

Si tous les participants ont une même conception de cette tâche, ils orientent leur description en fonction de leur propre activité et des tâches spécifiques qu'ils ont en charge. Les commerciaux insistent plus particulièrement sur la partie «interaction avec le client » alors que les contrôleurs décrivent plus précisément ce qui concerne la réglementation. Dans l'ensemble, les commerciaux s'avèrent plus loquaces que les contrôleurs sur la décomposition de la tâche d'EER en sous-tâches, ce qui semble naturel dans la mesure où ce sont eux qui ont en charge la plus grande partie de cette activité. Les contrôleurs citent davantage de sous-tâches liées au respect de la législation qu'ils n'en citent sur l'aspect interaction avec le client. En revanche, cette distinction n'est pas aussi marquée pour les commerciaux : ils citent quasiment autant de sous-tâches concernant l'aspect 
interaction avec le client, que de sous-tâches en lien avec le respect de la législation.

\subsubsection{Analyse des besoins d'information}

Les exemples de manques d'information qui peuvent apparaître lors de la réalisation de la tâche d'EER sont exprimés sous la forme de questions ou de thèmes. Leur analyse met en évidence plusieurs points :

○ Les besoins d'information dans l'activité quotidienne des interviewés prennent une place importante, pour toutes les soustâches composant l'EER. Il y a donc nécessité pour les employés de disposer de sources d'information leur permettant de trouver rapidement des réponses à leurs questions ;

o Chaque sous-tâche de l'EER génère des types de questions particulières ;

○ Des connaissances plus larges et non spécifiques à la tâche en cours sont attendues.

\begin{tabular}{|l|l|l|}
\hline \multicolumn{1}{|c|}{ Sous tâche } & Fonction & \multicolumn{1}{|c|}{$\begin{array}{c}\text { Exemples de besoin } \\
\text { d'information }\end{array}$} \\
\hline $\begin{array}{l}\text { Présentation des produits, de } \\
\text { 'entreprise }\end{array}$ & Commercial & $\begin{array}{l}\text { Fluctuation des taux, } \\
\text { Placements à court terme. }\end{array}$ \\
\hline $\begin{array}{l}\text { Présentation des règles de } \\
\text { fonctionnement de l'ouverture de } \\
\text { compte, des produits... }\end{array}$ & Commercial & $\begin{array}{l}\text { Quels sont les moyens de paiement } \\
\text { possibles pour un compte 18-20 } \\
\text { ans? }\end{array}$ \\
\hline $\begin{array}{l}\text { Demande d'informations } \\
\text { générales au client : } \\
\text { ses projet,, sa situation }\end{array}$ & Commercial & $\begin{array}{l}\text { Quelles informations demander au } \\
\text { client lors d'une ouverture de } \\
\text { compte ? }\end{array}$ \\
\hline $\begin{array}{l}\text { Demande de pièces justificatives } \\
\text { au client }\end{array}$ & Commercial & $\begin{array}{l}\text { Quel justificatif de domicile } \\
\text { fournir pour les non-résidents ? } \\
\text { Qui peut être admis comme } \\
\text { représentant légal pour un mineur } \\
\text { dont les parents ne résident pas en } \\
\text { France ? }\end{array}$ \\
\hline $\begin{array}{l}\text { Vérification des pièces } \\
\text { justificatives }\end{array}$ & Contrôleur & $\begin{array}{l}\text { Quels sont les justificatifs de } \\
\text { domicile admis? } \\
\text { Quelles sont les conditions } \\
\text { d'admissibilité des justificatifs ? }\end{array}$ \\
\hline Vérification du dossier complet & Contrôleur & $\begin{array}{l}\text { Quelles sont les conditions } \\
\text { d'admissibilité des justificatifs }\end{array}$ \\
\hline
\end{tabular}

Tableau 1 : Types de demandes d'information par sous-tâche

Le tableau ci-dessus récapitule, pour chaque sous-tâche de la tâche d'EER, des exemples de besoins d'information directement liés à l'EER. A chaque étape correspondent donc plusieurs types de demandes d'information. Elles peuvent être 
catégorisées de la manière suivante : un employé de la banque peut avoir besoin

o de connaissances procédurales ou de connaissances descriptives,

o d'informations ponctuelles, immédiates ou non,

o de connaissances spécifiques au domaine bancaire mais aussi de connaissances non spécialisées tout aussi importantes pour réaliser la tâche assignée.

Les connaissances procédurales peuvent s'exprimer de la manière suivante : «comment faire pour? » :

o réaliser une ouverture de compte imposée, ouvrir un compte pour les non-résidents, ouvrir un compte pour les gens du voyage, ...

Les connaissances descriptives peuvent concerner des produits : - Quelle est la fiscalité des Codevi ?, Comment a évolué la rémunération du PEL?

ou des dispositions réglementaires :

o Loi Scrivner, Fiscalité.

Quel que soit le type de connaissances concernées, ces besoins peuvent être immédiats. Dans ce cas, l'employé a besoin de cette information pour mener à bien la tâche ou sous-tâche dans laquelle il est engagé. La contrainte de temps est souvent très forte car l'information recherchée est indispensable pour que la tâche dans laquelle l'employé est engagé puisse aboutir, pour qu'une décision soit prise, un problème résolu. Mais ces besoins d'information peuvent aussi se situer en toile de fonds de l'activité (et/ou de chaque sous-tâche). Dans ce cas, l'information attendue permet une mise à jour des connaissances. L'employé ne cherche pas de l'information pour résoudre un problème immédiat et dans ce cas, le facteur temps semble jouer un rôle moins important. En revanche, l'information attendue doit permettre à l'utilisateur de compléter ses connaissances pour effectuer d'une manière plus satisfaisante son activité quotidienne. Grâce à l'information obtenue, l'utilisateur (surtout quand il est expert) peut même anticiper sur d'éventuelles situations futures, et amorcer d'autres recherches d'information. 
Cette première analyse permet plusieurs constats : d'abord, la variété des besoins d'information susceptibles de poser problème dans la pratique de l'activité professionnelle; et ensuite le fait que, même pour une tâche qui semble clairement délimitée et même routinière de l'avis de certains sujets, les interviewés sont amenés à s'interroger sur des points non directement liés à la tâche qu'ils ont à effectuer. Par exemple, ils cherchent de l'information sur les produits, les assurances, la fiscalité,... mais ils peuvent aussi s'interroger sur le fonctionnement du système informatique utilisé pour l'ouverture de compte, les accès à telle ou telle fonctionnalité.

On remarque également l'importance de l'information et de la recherche d'information dans l'activité des collaborateurs de la banque, et ce quelle que soit leur fonction, mais sans noter d'influence particulière de l'expertise sur la nature de l'information attendue.

Enfin, il est clair que les individus enquêtés ne voient pas la recherche d'information comme un aspect distinct de leur activité quotidienne, confirmant ainsi d'autres travaux (Jarvelin, 2004) : la recherche d'information et les échanges d'informations sont imbriqués dans l'activité principale. D'ailleurs, Guyot (Guyot 2002) estime que «l'informationobjet » double l'activité principale qui, à son tour, lui donne son contexte et ses caractéristiques. En même temps, l'activité d'information constitue une activité en elle-même, activité complexe tant dans sa forme (rechercher de l'information, informer les autres, s'informer soi-même..) que dans sa mise en œuvre (mobiliser des moyens, développer des capacités pour conduire les tâches qui composent chaque opération).

\section{2-2-2- L'influence de l'expertise sur les stratégies d'accès à l'information}

Un grand nombre de sources d'information sont disponibles pour les employés de l'entreprise. Le tableau 2 présente la liste des sources d'information mentionnées par les interviewés et le nombre de fois où chaque source a été mentionnée. 


\begin{tabular}{|l|c|c|c|c|c|}
\hline & $\begin{array}{c}\text { Comm. } \\
\text { novice }\end{array}$ & $\begin{array}{c}\text { Comm } \\
\text { expert }\end{array}$ & $\begin{array}{c}\text { Contr. } \\
\text { novice }\end{array}$ & $\begin{array}{c}\text { Contr } \\
\text { expert }\end{array}$ & Moyenne \\
\hline $\begin{array}{l}\text { Documents de } \\
\text { référence }\end{array}$ & $50 \%$ & $66 \%$ & $100 \%$ & $100 \%$ & $79 \%$ \\
\hline $\begin{array}{l}\text { Collègues, } \\
\text { forums internes }\end{array}$ & $75 \%$ & $66 \%$ & $100 \%$ & $40 \%$ & $70,2 \%$ \\
\hline $\begin{array}{l}\text { Formations } \\
\text { séminaires }\end{array}$ & $50 \%$ & $33 \%$ & $100 \%$ & $40 \%$ & $55,7 \%$ \\
\hline Fiches & $100 \%$ & $100 \%$ & $0 \%$ & $0 \%$ & $50 \%$ \\
\hline Courriels & $50 \%$ & $100 \%$ & $0 \%$ & $20 \%$ & $42,5 \%$ \\
\hline $\begin{array}{l}\text { Campagnes } \\
\text { d'inform. }\end{array}$ & $0 \%$ & $66 \%$ & $0 \%$ & $40 \%$ & $26,5 \%$ \\
\hline Internet & $25 \%$ & $33 \%$ & $0 \%$ & $0 \%$ & $14,5 \%$ \\
\hline Revues & $25 \%$ & $33 \%$ & $0 \%$ & $0 \%$ & $14,5 \%$ \\
\hline
\end{tabular}

Tableau 2 : Liste des sources d'information.

Les «documents de référence » (ligne 1 du tableau) sont des documents «sources » pour la banque. Ils décrivent de manière précise et détaillée les produits, les procédures à exécuter. Ils ne s'adressent pas à une catégorie d'employés en particulier mais à l'ensemble du personnel de la banque et ont pour vocation d'être accessibles et exploitables par tous les employés, quelque soit leur fonction. Les «fiches » (ligne 3 du tableau) sont conçues spécifiquement pour les commerciaux. Elles sont synthétiques (un recto verso maximum) et présentent de manière rapide les points-clés d'une activité ou d'un produit.

Le tableau ci-dessus montre la diversité des 8 sources d'information citées par les sujets : certaines sont sur support papier, d'autres sur support électronique, certaines sont formalisées, d'autres sont informelles. La plupart des sujets s'accordent à dire qu' "il y en a même trop ». L'un des interviewés souligne qu'en ce qui concerne la gestion de l'information à la banque «ça coince quelque part», sans pouvoir préciser plus clairement ce qui pose problème.

Tous sujets confondus, les «documents de référence» sont les sources d'information les plus citées. Les collaborateurs de la banque, quelle que soit leur fonction, estiment effectivement que 
ces documents sont riches d'informations. Pourtant, ces documents sont très inégalement utilisés. D'une façon générale, les sujets s'accordent pour dire que la consultation des ces documents est difficile. Les personnes qui consultent régulièrement les documents de référence préfèrent les imprimer pour travailler et y revenir éventuellement plus tard. Pour des recherches précises, ils utilisent la base de données donnant accès à ces documents. Plusieurs raisons sont mentionnées pour expliquer l'utilisation inégale des documents de référence: beaucoup de sujets considèrent qu'il faut des connaissances et de l'expérience pour arriver à comprendre, interpréter et exploiter l'information contenue dans les «documents de référence». De ce fait, il apparaît que ce sont les utilisateurs les plus expérimentés qui utilisent le plus régulièrement ces documents. Le vocabulaire qui y est utilisé est qualifié de «hermétique ». Enfin, la fréquence de consultation des «documents de référence » dépend des fonctions assumées par les collaborateurs de la banque. Ainsi, les contrôleurs, quelque soit leur niveau d'expertise, sont ceux qui consultent le plus souvent ce type de documents.

Après les «documents de référence », c'est le recours aux collègues qui est privilégié, via le téléphone, un courriel ou en postant un message sur un forum interne. La rapidité de la réponse est une des motivations principales de ce choix.

Ce sont les novices qui citent le plus ce moyen d'accès à l'information. Pour des questions précises, ces employés préfèrent demander d'abord à un collègue plus expérimenté, avant d'éventuellement opter pour une recherche dans des sources formelles.

La ventilation par métiers met en évidence le fait que les commerciaux, quelque soit leur niveau d'expertise, citent en priorité «les fiches », ce qui logique puisque «les fiches» ont été conçues spécifiquement pour cette catégorie d'employés, pour aider à vendre des produits. Dans un second temps, ce sont les sources non formelles qui sont mentionnées. Ici, les novices s'adressent directement à leurs collègues alors que les experts consultent leur «réserve» de mails jugés importants et provenant des collègues, ou des services de la banque et présentant des nouvelles procédures ou nouveaux produits. 
Cette « réserve », souvent organisée par dossiers thématiques, leur permet de retrouver rapidement l'information en temps utiles. Une proportion plus importante de commerciaux experts cite les «documents de référence»; ces documents sont effectivement plus faciles d'accès pour les individus ayant une certaine expérience dans la banque et une connaissance du vocabulaire spécialisé utilisé dans ces documents.

Les contrôleurs recherchent en premier lieu l'information dont ils ont besoin dans les «documents de référence ». Ils citent ensuite la formation et les séminaires comme vecteur d'information à égalité avec le recours aux personnes ressources. Là aussi, le niveau d'expertise semble un facteur essentiel. Ainsi, pour les experts, les «documents de référence » apparaissent clairement comme la source principale. En revanche, pour les novices, les «documents de référence »sont cités à égalité avec le recours aux collègues et les séances de formation. Le contrôleur expert a recours à ses collègues de manière à vérifier une information ou pour « revérifier» une réponse déjà obtenue ; pour eux, les formations et séminaires apparaissent comme des sources d'informations transmises par d'autres experts de l'entreprise.

L'utilisation des ressources formelles est réelle mais elle est plutôt choisie par les employés les plus experts. Leur habitude du contenu de ces documents mais aussi des modalités pour y accéder (système d'information de l'entreprise) leur permettent d'avoir recours à ces ressources sans perdre de temps et en étant assurés des réponses obtenues. On note toutefois l'importance de la place des ressources informelles dans le processus d'accès l'information. Le recours aux collègues est général, mais on remarquera qu'on ne s'adresse pas seulement au plus expert de l'entreprise, mais de préférence à quelqu'un que l'on connaît et à qui on fait confiance.

Devant le nombre et la diversité des informations, beaucoup de collaborateurs tendent à se constituer des dossiers personnels d'information. Ces dossiers regroupent différents types de documents : fiches, mails, pages web, documents de référence traitant d'un cas ou d'une activité précise. Ceci nous rapproche $\mathrm{du}$ concept de «personal information management» (Bruce, 
2005). Selon lui, une des activités clé de cette gestion de l'information est de retenir l'information pour une utilisation et un accès futur. Les individus conservent ainsi de l'information parce qu'ils pensent qu'elle pourra leur être utile plus tard. Ils fondent cette attitude de conservation sur une anticipation de leurs besoins d'information. Cette manière de procéder permet aux individus de faire face à l'abondance d'information. Ils ont accès à plus d'information qu'ils ne peuvent en traiter ; et cette information joue un rôle fondamental dans l'activité professionnelle. Cette collecte personnelle d'information peut prendre diverses formes: articles, favoris, mails, carnets d'adresses,.... Le recours à ce type de ressources est perçu comme sécurisant parce que l'on peut les garder «sous le coude » qu'ils soient sur papier ou sur le poste de travail.

\section{2-2-3- Les contraintes et enjeux de la recherche d'information liés à la fonction et à l'expertise}

Si l'activité d'information est une activité secondaire et totalement imbriquée dans l'activité principale de l'individu, il semble que les objectifs liés à l'activité principale influencent les pratiques d'information et notamment les stratégies d'accès à l'information. La mise en relation des pratiques d'information avec la fonction et le niveau d'expertise montre que les sujets mettent en œuvre des stratégies différentes.

Dans une organisation ou une entreprise, lorsqu'un commercial doit effectuer un service au public, comme l'accueil ou comme l'orientation vers le bon service, il peut être amené à devoir rechercher une information pour accomplir son travail. Le commercial se trouve alors dans une situation paradoxale :

○ d'un côté, il accueille le client vis-à-vis duquel l'objectif est de type relationnel : il faut lui faire bon accueil et se montrer efficace pour le service demandé, sachant que dans le cas contraire, le client, l'usager pourrait se retourner vers un concurrent ou un autre service ;

○ de l'autre, il rend compte à sa hiérarchie ; ici, l'objectif serait du type commercial puisqu'il s'agit d'obtenir des résultats commerciaux, décrocher des contrats par exemple, empêcher que le client ne s'échappe vers les concurrents. Mais le commercial se doit également de conduire son activité avec 
prudence et dans un cadre réglementaire très strict, sous peine de mettre son entreprise en situation illégale.

L'activité de recherche d'information du commercial s'inscrit donc dans cette situation paradoxale :

- Côté client, l'employé qui prend du temps pour vérifier une information, ou faire confirmer un choix par un collègue risque d'apparaître comme méfiant sinon peu efficace, voire même comme incompétent aux yeux du client dans la mesure où il ne fournit pas immédiatement la réponse au problème soulevé.

- Côté hiérarchie, il pourra apparaître, au contraire, comme un employé prudent et responsable. En effet, il montre qu'il a à cœur de ne pas engager son entreprise dans une situation potentiellement difficile.

Les contrôleurs, eux-aussi peuvent se trouver dans une situation paradoxale :

○ Dans leur activité principale, ils ont un objectif de vérification en vue du respect de la légalité. En effet, ce sont eux qui s'assurent que les procédures d'ouverture de comptes respectent les règles légales et ils doivent en rendre compte à leur hiérarchie.

- Mais ils sont d'un autre côté confrontés aux commerciaux puisqu'ils valident et vérifient le travail fourni par ces derniers.

La recherche d'information des contrôleurs s'inscrit dans ce paradoxe :

○ Le contrôleur doit être suffisamment prudent pour assurer à sa hiérarchie qu'il ne laissera pas passer un dossier « risqué », - mais s'il est trop pointilleux dans sa vérification, il peut apparaitre méfiant et suspicieux par rapport au travail de ses collègues commerciaux.

Les stratégies d'accès à l'information ne sont pas régulières : certains individus privilégient des stratégies individuelles, opportunistes pour s'informer. A ce niveau, l'expertise de l'employé conduit alors à des pratiques différentes. Ainsi, dans une perspective de prudence et de responsabilité, un novice aura tendance à rechercher l'approbation de collègues plus compétents que lui, en téléphonant au service juridique, en envoyant un mail par exemple. Occupé à se former et à acquérir 
les connaissances de base, le novice exploite les sessions de formations internes, mais il a moins tendance à explorer la documentation sur place, à aller sur la Toile chercher des informations parce qu'il ne connaît pas les ressources pertinentes et parce que ses connaissances ne sont suffisantes pour évaluer la fiabilité des informations récoltées.

Les plus experts privilégient non seulement la consultation des sources formelles, (banques de données internes ou externes; portails spécialisés, etc.), mais aussi la mise en place de véritables stratégies de mises à jour de leurs connaissances, par exemple par la constitution de dossiers personnels, sous forme papier ou électronique (Jones, 2004) (Bruce, 2005) (Accart, 2002). Les informations réunies dans ces dossiers personnels permettent à la fois de répondre aux clients mais aussi aux collègues, notamment les plus nouveaux. Ainsi, on tend à conserver non seulement des documents sur les produits bancaires du jour, mais également sur des produits plus anciens pour répondre aux questions du genre "quel était de taux du PEL il y a 10 ans ? ». De la même manière, on connaît les sites utiles et spécialisés sur la toile ; ils sont réunis dans des favoris très facilement accessibles. Toutes ces informations permettent d'anticiper les questions des uns et des autres : à partir du moment où une question est posée, qu'elle vienne d'un client ou d'un collègue, on veut être capable d'y répondre. Dans un contexte de compétition, de conflits d'intérêts, on veut éviter que le demandeur s'adresse à quelqu'un d'autre. Si pour le demandeur, l'objectif est de diminuer une incertitude, il s'agit pour le «détenteur » de s'affirmer comme capable de trouver ou de détenir des informations. La «maîtrise de l'information » devient alors un symbole de compétence, une marque d'efficacité. C'est un des moyens permettant à l'expert de se positionner à l'intérieur de son périmètre d'action et de mesurer sa place dans l'activité générale (March, 1991 cité par Vacher, 2006).

\section{Conclusion}

Cette étude menée au sein d'une entreprise bancaire montre comment le contexte et l'activité principale des employés de la banque influent sur leurs besoins d'information 
ainsi que sur les stratégies mises en œuvre pour l'accès à l'information. Le niveau d'expertise est un facteur induisant des pratiques différentes notamment dans le choix des sources d'information. L'étude montre enfin que l'activité d'information dépasse, pour les employés, la simple récupération de l'information. Plus que jamais, les stratégies d'accès à l'information représentent un enjeu stratégique pour l'image de l'individu au sein de l'entreprise.

\section{Références}

Accart JP., Peyrelong MF. 2002. Du système d'information personnel au système d'information collectif : réalités et mirages du partage de l'information en entreprise. Actes de la conférence de l'association canadienne des sciences de l'information CAIS/ACSI, Toronto, 2002.

Bartlett J., Toms E. 2005. How is Information Used? Applying task analysis to understanding information use, Actes de la conference ACSI/CAIS, Londres, 2005.

Bergeron P. 2007. Vers une gestion de l'information "2.0"? Maximiser la collaboration entre les fonctions d'information pour améliorer les capacités informationnelles dans l'organisation. Présentation orale, GTEC, Ottawa, 15 octobre 2007. [en ligne] consulté le 18/11/2008. http://www.tbssct.gc.ca/im-gi/imday07jourgi/pres/bergeron-fra.pdf

Bruce H. 2005. "Personal, anticipated information need". Information research, vol 10, $\mathrm{n}^{\circ} 3$, Avril 2005. [en ligne]

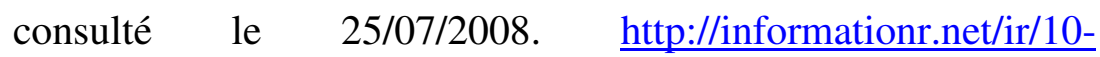
3/paper232.html

Cheuk W. 1999. "The derivation of a "situational" information seeking and use process model in the workplace: employing 
sense-making". Paper presented at International Communication Association annual meeting, San Francisco, California.

Choo W. 2007."Information seeking in organizations: epistemic contexts and contests“. Information Research, vol. 12, $\mathrm{n}^{\circ} 2$, January 2007.

Dinet, J., Rouet, J.-F. 2002. La recherche d'information : processus cognitifs, facteurs de difficultés et dimension de l'expertise. In C. Paganelli (Ed.), Interaction homme - machine et recherche d'information (pp.133-161). Paris : Hermès.

Ericsson KA., Charness, N. 1997. Cognitive and developmental factors in expert performance, in Feltovich, PJ., Ford, KM., and Hoffman, RR., (eds), Expertise in Context: Human and Machine, AAAI Press/The MIT Press, Menlo Park, CA, 1-41, 1997.

Guyot B. 2000. Les dynamiques informationnelles, Note de présentation de travaux en vue de l'HDR en sciences de l'information et de la communication, Université Stendhal, Grenoble 3, 130p, 2000.

Guyot B. 2002. «Une activité de travail méconnue : l'activité d'information », colloque ICUST usages, 2002.

Hung SY. 2003. Expert versus novice use of the executive support systems: an empirical study. Information and Management, 40, pp1777-189, 2003;

Järvelin K., Ingwersen P. 2004. "Information seeking research needs extension towards tasks and technology" Information Research, 101 paper 212. [en ligne] consulté le 31/07/2008. http://InformationR.net/ir/10-1/paper212.html 
Jones W. 2004. "Finders, keepers : the present and future perfect in support of personal information management". First Monday, 93. [en ligne] consulté le 27/07/2008. http://www.firstmonday.org/issues/issue9_3/jones/index.html Kulthau, C. 2004. "Towards collaboration between information seeking and information retrieval." Information Research, vol. 10, no 2, January 2005 Papers presented at ISIC 2004: the 5th Information Seeking in Context Conference, Dublin, Ireland, 13 September, 2004. [en ligne] consulté le 07/03/2009. http://informationr.net/ir/10-2/paper225.html

Marchionnini G., Lin X., Dwiggins S. 1990. "Effects of search and subject expertise on information seeking in a hypertext environment" in D. Henderson (dir.), Proceedings of the American Society for Information Science, annual meeting, Learned Information, Toronto, (pp.129-142), 1990.

Olmstadt, W. 2000. Cataloging Expert Systems: Optimism and Frustrated Reality. Journal of Southern Academic and Special Librarianship: 01 (2000).

Paganelli C., Mounier E., 2003. "Information Retrieval in Technical Documents: from the User's query to the Information-Unit Tagging”. SIGDOC'03, San Francisco, October 2003.

Vacher B. 2006. Oubli, étourderie, ruse et bricolage organisés : arrêt sur théories, in Communication, sens et intersubjectivité en organisation: aperçus de la réflexion actuelle dans la francophonie dir. par Sylvie Grosjean et Luc Bonneville, 2006. 